Intestinal obstruction caused by phytobezoar composed of jaboticaba seeds: case report and literature review

Rafael Luís Luporini, Lícia Deon Weirich, Marcelo Rodolfo Marciano, Luiz Vagner Sipriani, Francisco de Assis Gonçalves Filho, Anwar Fausto Félix Sabbag, Marcelo Maia Caixeta Melo, Luís Sérgio Ronchi, Geni Satomi Cunrath, João Gomes Netinho .308

\section{Actinomycosis mimicking colonic neoplasia}

Luísa Lima Castro, Mônica Maria Demas Álvares Cabral, Rafael Felipe Maciel Andrade, Kelly Cristine de Lacerda Rodrigues Buzatti, Rodrigo Gomes da Silva. 312

Giant desmoid tumor of the abdominal wall in a patient with Gardner Syndrome

Daniel Paulino Santana, Juliano Alves Figueiredo, Matheus Matta Machado Mafra Duque Estrada Meyer, Paula Mendonça Pimenta Ferreira, Guilherme Sousa Sarmento Valente, Marcos Wanderley Campos Reis .316

Giant ileal inflammatory fibroid polyp: a rare cause of intestinal intussusception

Daniel Cury Ogata, Elisiário Pereira Neto, Daniel Oséias Sezerino, Joel Antônio Bernhardt 321
Appendiceal endometriosis as a rare cause of abdominal pain a case report and literature review

Rafael Denadai, Rafael Aliceda Ferraz, Ricardo De Álvares

Goulart, Rogério Saad-Hossne, Fábio Vieira Teixeira 324

\section{SPECIAL SESSION}

Association between celiac disease and Chron's disease - a challenge to the coloproctologist

Leonardo Estenio Iezzi, Bruno Amaral Medeiros, Marley Ribeiro Feitosa, Ana Luiza Normanha Ribeiro De Almeida, Rogério Serafim Parra, José Joaquim Ribeiro Da Rocha, Omar Feres...329

Can statins improve outcome in colorectal surgery? Part I Júlio César M. Santos JR

ACCREDITED SERVICES .339

\title{
ERRATUM
}

J Coloproctol. 2012;32(2):103.

Instead of:

Campos FGCM, Silva GJS. In memoriam - José Hyppolito da Silva (1938-2011). J Coloproctol, 2012;32(2): 103-105.

Insert:

Campos FGCM, Formiga GJS. In memoriam - José Hyppolito da Silva (1938-2011). J Coloproctol, 2012;32(2): 103-105. 